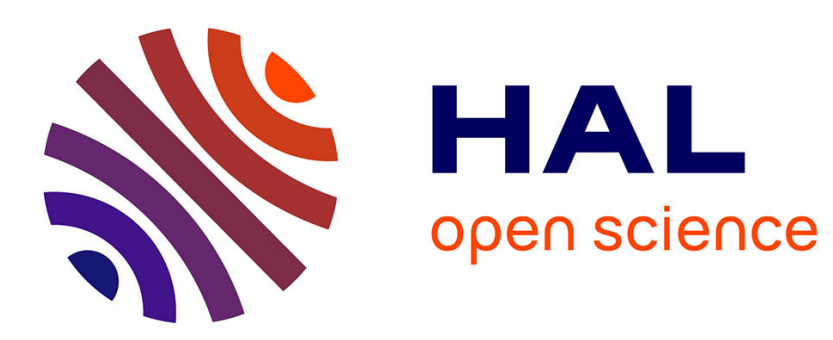

\title{
The evolution of social learning rules: Payoff-biased and frequency-dependent biased transmission
}

\author{
Jeremy Kendal, Luc-Alain Giraldeau, Kevin Laland
}

\section{To cite this version:}

Jeremy Kendal, Luc-Alain Giraldeau, Kevin Laland. The evolution of social learning rules: Payoffbiased and frequency-dependent biased transmission. Journal of Theoretical Biology, 2009, 260 (2), pp.210. 10.1016/j.jtbi.2009.05.029 . hal-00554619

\section{HAL Id: hal-00554619 https://hal.science/hal-00554619}

Submitted on 11 Jan 2011

HAL is a multi-disciplinary open access archive for the deposit and dissemination of scientific research documents, whether they are published or not. The documents may come from teaching and research institutions in France or abroad, or from public or private research centers.
L'archive ouverte pluridisciplinaire HAL, est destinée au dépôt et à la diffusion de documents scientifiques de niveau recherche, publiés ou non, émanant des établissements d'enseignement et de recherche français ou étrangers, des laboratoires publics ou privés. 


\section{Author's Accepted Manuscript}

The evolution of social learning rules: Payoff-biased and frequency-dependent biased transmission

Jeremy Kendal, Luc-Alain Giraldeau, Kevin Laland

PII: S0022-5193(09)00228-8

DOI: doi:10.1016/j.jtbi.2009.05.029

Reference: YJTBI 5558

To appear in: $\quad$ Journal of Theoretical Biology

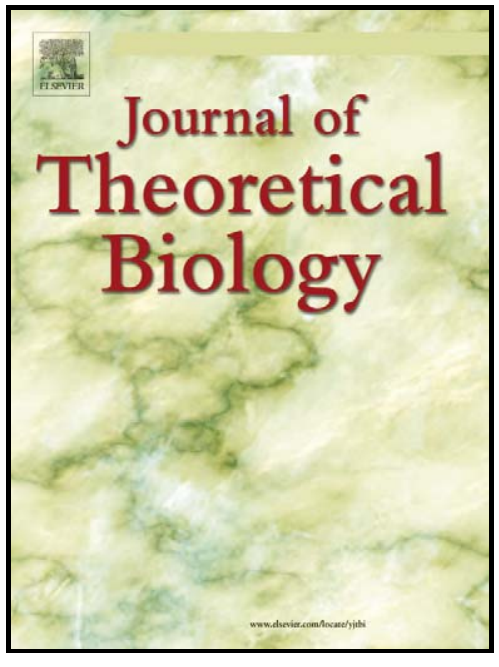

www.elsevier.com/locate/yjtb

Received date: $\quad 7$ November 2008

Revised date: $\quad 8$ May 2009

Accepted date: $\quad 8$ May 2009

Cite this article as: Jeremy Kendal, Luc-Alain Giraldeau and Kevin Laland, The evolution of social learning rules: Payoff-biased and frequency-dependent biased transmission, Journal of Theoretical Biology, doi:10.1016/j.jtbi.2009.05.029

This is a PDF file of an unedited manuscript that has been accepted for publication. As a service to our customers we are providing this early version of the manuscript. The manuscript will undergo copyediting, typesetting, and review of the resulting galley proof before it is published in its final citable form. Please note that during the production process errors may be discovered which could affect the content, and all legal disclaimers that apply to the journal pertain. 


\title{
The Evolution of Social Learning Rules:
}

\section{payoff-biased and frequency-dependent biased}

\section{transmission}

\author{
Jeremy Kendal ${ }^{\mathrm{a}, *}$ \\ ${ }^{a}$ Department of Anthropology, University of Durham, Dawson Building, South \\ Road, Durham, DH1 3LE, U.K. \\ Luc-Alain Giraldeau ${ }^{\mathrm{b}}$ \\ b Département des sciences biologiques, Université du Québec à Montréal, \\ Montréal HЗС 3P8, Canada \\ Kevin Laland ${ }^{\mathrm{c}}$ \\ 'School of Biology, University of St. Andrews, Bute Medical Building, Queen's \\ Terrace, St. Andrews, Fife, KY16 9TS, U.K.
}

\begin{abstract}
Humans and other animals do not use social learning indiscriminately, rather, natural selection has favoured the evolution of social learning rules that make selective use of social learning to acquire relevant information in a changing environment. We present a gene-culture coevolutionary analysis of a small selection of such rules (unbiased social learning, payoff-biased social learning and frequency-dependent biased social learning, including conformism and anti-conformism) in a population of asocial learners where the environment is subject to a constant probability of
\end{abstract}


change to a novel state. We define conditions under which each rule evolves to a genetically-polymorphic equilibrium. We find that payoff-biased social learning may evolve under high levels of environmental variation if the fitness benefit associated with the acquired behaviour is either high or low but not of intermediate value. In contrast, both conformist and anti-conformist biases can become fixed when environment variation is low, whereupon the mean fitness in the population is higher than for a population of asocial learners. Our examination of the population dynamics reveals stable limit cycles under conformist and anti-conformist biases and some highly complex dynamics including chaos. Anti-conformists can out-compete conformists when conditions favour a low equilibrum frequency of the learned behaviour. We conclude that evolution, punctuated by the repeated successful invasion of different social learning rules, should continuously favour a reduction in the equilibrium frequency of asocial learning, and propose that, among competing social learning rules, the dominant rule will be the one that can persist with the lowest frequency of asocial learning.

Key words: cultural evolution, gene-culture coevolution, social learning, conformity, social learning strategy

\footnotetext{
* Corresponding Author

Email address: jeremy.kendal@durham.ac.uk

Tel. +44 (0) 191334 6130; Fax. +44 (0) 1913341615 (Jeremy Kendal).
} 


\section{Introduction}

Social learning denotes the transmission of learned information from one individual to another and can occur by a number of mechanisms including enhancement effects, imitation and emulation (Galef and Giraldeau, 2001; Whiten and Ham, 1992). Conversely, asocial (or individual) learning occurs independently of any social influence. Historically, scientists have tended to assume that individuals should rely on social learning when they can, but recent mathematical analyses reveal that this is incorrect, and that some mixture of social and asocial learning is expected to occur in a changing environment (Boyd and Richerson, 1985; Rogers, 1988; Feldman et al., 1996; Henrich and McElreath, 2003; Laland, 2004; Enquist et al., 2007). This result derives from a trade-off between the benefits of asocial and social learning that, as pointed out by Kameda and Nakanishi (2002) and Laland (2004), is similar to the producer-scrounger dilemma found in social foragers (Barnard and Sibly, 1981; Giraldeau and Caraco, 2000). Asocial learners (information producers) typically incur additional temporal or energetic costs as well as risk of mortality or injury associated with learning from direct interaction with the environment. While social learners (information scroungers) can acquire information relatively cheaply (i.e. they are free-riders), they are more liable than asocial learners to acquire outdated information that has no associated fitness benefit in a changing environment (equivalent to not receiving a finder's advantage, that is, the exclusive payoff enjoyed by the information producer). Consequently, social learning is thought to evolve to a polymorphic equilibrium at least under intermediate levels of environmental variation and in more stable environments than those favouring asocial learning alone (Aoki et al., 
2005).

Rogers (1988) showed that the fitness of social learners at the polymorphic equilibrium would be no greater than the average individual fitness in a population of asocial learners, assuming asocial learning fitness is constant (or independent of social learning frequency) and that social learners' only benefit over asocial learners was cost-free acquisition of information. When rare, the fitness of social learners exceeds that of asocial learners, but declines with frequency as there are fewer asocial learners producing adaptive information in a changing environment. The population evolves to a mixed evolutionarily stable strategy (ESS) where, by definition, the fitnesses of social learners equates to that of asocial learners (Giraldeau et al., 2002; Henrich and McElreath, 2003). Rogers assumed that social learners were unbiased in their choice of whom to copy and thus the probability of social learning of a behaviour was proportional to its frequency in the population.

Boyd and Richerson (1995) suggested that Rogers' (1988) result was paradoxical in that it contrasts with a commonly held assertion that culture enhances fitness. Although Rogers' result is not inherently paradoxical, it appears to conflict with the observation that social learning underlies the effect of human culture on our ecological success and population growth. One resolution to this conundrum is to recognise that, in a changing environment, selection ought to have fashioned in our minds specific evolved rules (Boyd and Richerson, 1985), or 'social learning strategies' (Laland, 2004), that specify the circumstances under which individuals should exploit information from others, and from whom they should learn. A small number plausible strategies have been subject to theoretical analysis (e.g. Boyd and Richerson (1985); Henrich and McElreath (2003); Laland (2004)) and some have received experimental sup- 
port (Coolen et al., 2003; Kendal et al., 2005; Galef and Whiskin, 2008). For instance, Giraldeau and Lefebvre (1987) argued that pigeons failed to copy tutors because the tutors were apparently obtaining a smaller reward than their own (i.e. payoff-biased social learning).

Compared to a population composed entirely of asocial learners, the average individual fitness at equilibrium is enhanced if individuals exhibit cognitive flexibility, switching between asocial and social learning in a noisy two-state environment (Boyd and Richerson, 1995; Kameda and Nakanishi, 2003). Similarly, Enquist et al. (2007) showed that a strategy of 'critical social learning', where individuals only adopt asocial learning if social learning is unsatisfactory, also enhances average fitness at equilibrium. Boyd and Richerson (1995) also showed that average fitness is higher than that in a population of asocial learners if social learners can improve their learned behaviour so that there is cumulative cultural evolution.

Social transmission biases can be classified into context bias, including frequencydependent biases, and content bias, including payoff-bias (Boyd and Richerson, 1985; Henrich and McElreath, 2003). Frequency-dependent bias refers to a non-linear relationship between the frequency of a behaviour and its probability of adoption, in particular, conformity and anti-conformity (sometimes referred to as 'non-conformity'). Wakano and Aoki (2007) and Nakahashi (2007) both found a negative relationship between the evolutionarily stable strength of conformist bias and environmental stability (contrary to Henrich and Boyd (1998)), while Nakahashi (2007) also found a negative relationship with the cost of asocial learning. However, these studies do not explore the full range of frequency-dependent bias (i.e. from anti-conformist to conformist). 
Payoff-biased transmission assumes that the probability of adoption is directly related to characteristics of the observed behaviour (Boyd and Richerson, 1985). Using an optimality approach to compare social learning rules in a multi-armed bandit setting, Schlag (1998) discovered that the most successful strategy was the 'proportional imitation rule', where an individual copies a demonstrator that received a higher payoff than their own with a probability that is proportional to the difference in payoffs (approximating the replicator dynamic).

It is commonly assumed that learning and biological evolution operate at different timescales, where natural selection acts on a stationary distribution of cultural traits (Boyd and Richerson, 1985, 1995; Enquist et al., 2007). This assumption is often employed for mathematical convenience, yet in reality, cultural dynamics can take many generations to reach a stable state (CavalliSforza and Feldman, 1981; Boyd and Richerson, 1985) while biological evolution can be fast (Kingsolver et al., 2001). Moreover, the trajectory of genetic evolution may be influenced by the frequency of the learned trait prior to equilibrium. In these cases, a gene-culture coevolutionary approach is appropriate to address the coevolution of learned behaviour and social learning rules (Henrich and Boyd, 1998; Nakahashi, 2007; Wakano and Aoki, 2007).

The present study is motivated by the need for a fully coevolutionary analysis of the evolution of social learning rules, one that considers the full range of frequency-dependent biases, comparing these to other learning rules and with an environment subject to a constant probability of change to a novel state. First, as a benchmark, we consider the standard case of unbiased social learning, where the probability of offspring acquiring a learned behaviour is proportional to the frequency of that behaviour in the population. Second, we 
consider payoff-biased social learning, where the probability of social learning is proportional to the observed benefit received by 'demonstrator' individuals performing a learned behaviour. Lastly, we consider frequency-dependent biased social learning, where the probability of social learning is disproportionately large (conformist) or small (anti-conformist) when observed in the majority of the population, and the reverse when observed in the minority.

\section{The Model}

We assume an initial population of obligate asocial learners that pay a cost $c_{A}$ to acquire novel learned information (labelled 'type $1^{\prime}$ ), expressed in a corresponding type 1 behaviour, that confers a fitness benefit $b$. We assume that asocial learning is always successful, such that all asocial learners acquire type 1 information. We then consider the genetic evolution of a tendency for offspring to learn socially, at cost $c_{S}$, from behaviour observed in the parental generation (that is, oblique transmission). We assume that the cost of asocial learning is greater than that of social learning as experimenting directly with the environment can take time, energy and be dangerous. Also, we assume that the benefit of acquiring type 1 behaviour outweighs its cost, such that a population of asocial learners will not go extinct. In summary, $0<c_{S}<c_{A}<b$.

We assume a constant probability of environment change between generations, $e$, so that there is a probability $1-e$ that the type 1 behaviour will provide the same benefit to offspring that it did for the previous generation. We assume that each generation of asocial learners always learns behaviour that reaps the benefit $b$, and so does not suffer the possibility of acquiring irrelevant or outdated information. However, social learners that do not learn type 1 
behaviour, or learn but encounter a changed environment, receive no fitness benefit.

The constant between-generation probability of environmental change assumes that information discovered asocially in the grandparental generation has the same probability of remaining relevant upon social transmission to the parental generation as it has upon further transmission between parental and offspring generations. This assumption is appropriate to model circumstances where a proportion $(e)$ of the population experiences a perturbation in the environment for a single generation that renders previously useful information redundant and that, for each generation, there is the same probability of perturbation. Alternatively, one might consider that behaviour type 1 represents any one of a large number of equally adaptive behaviors, of which a fraction $e$ becomes outdated each generation. Both cases differ from the coevolutionary approach instigated by Feldman et al. (1996) whereupon an environmental perturbation renders all previously adaptive information redundant (this approach requires separate recursions for periods of environmental stasis and perturbation). Information producers are necessary for social learning to persist when subject to this type of environmental variation. Note that, like Feldman et al. (1996), the environment provides no constraint upon from whom a social learning offspring might learn (i.e. a mean-field approach).

Our model tracks the frequency, across discrete generations, of three types of individual, or 'phenogenotypes': asocial learners, $f_{A}$, social learners that successfully use behaviour type $1, f_{S 1}$ and social learners that do not successfully use behaviour type $1, f_{S 0}$. We assume fertility selection, through differential reproduction, followed by offspring learning, with haploid genetic inheritance, and where absolute genetic fitness (here, reproductive success) is $1-c_{A}+b$, 
$1-c_{S}+b$ and $1-c_{S}$ for individuals of type A, S1 and S0, respectively. Recursions giving the frequencies of each phenogenotype in terms of their frequencies in the previous generation are as follows:

$$
\begin{aligned}
& \bar{W} f_{A}^{\prime}=f_{A}\left(1-c_{A}+b\right) \\
& \bar{W} f_{S 1}^{\prime}=\left[f_{S 1}\left(1-c_{S}+b\right)+f_{S 0}\left(1-c_{S}\right)\right] \phi(1-e) \\
& \bar{W} f_{S 0}^{\prime}=\left[f_{S 1}\left(1-c_{S}+b\right)+f_{S 0}\left(1-c_{S}\right)\right][1-\phi(1-e)]
\end{aligned}
$$

where each recursion is normalized by the mean fitness for the population, $\bar{W}=1-c_{A} f_{A}-c_{S}\left(1-f_{A}\right)+b\left(f_{A}+f_{S 1}\right)$, so that $f_{A}^{\prime}+f_{S 1}^{\prime}+f_{S 0}^{\prime}=1$.

The expression within the square brackets of (2) represents the non-normalized offspring frequency of social learners, prior to offspring learning. The probability that social learners receive the benefit $b$ of behaviour type 1 is conditional on the probability of social learning, $\phi$, and the probability that the environment remains constant between sequential generations, $1-e$, where

$$
\phi=s\left[(1-D)\left(f_{A}+f_{S 1}\right)+D \sum_{k=n / 2+1}^{n}\left(\begin{array}{l}
n \\
k
\end{array}\right)\left(f_{A}+f_{S 1}\right)^{k} f_{S 0}{ }^{n-k}\right],
$$

$s \leq 1$ is the fidelity of social transmission and $D$ weights the frequencydependent bias. Here, $0<D \leq 1$ for conformists, $-1 \leq D<0$ for anticonformists, and $D=0$ for unbiased social learners. When $D=0$, the probability of learning type 1 behaviour is the proportion of type 1 individuals in the parental generation $\left(f_{A}+f_{S 1}\right)$, scaled by $s$; that is social learners are unbiased in their choice of model (Figure 1). Payoff-biased social learning is incorporated by assuming a fidelity of transmission $s_{P B}=b$, where $b \leq 1$. 
Following Efferson et al. (2008), we use the binomial distribution to model a frequency-dependent biased social learning, and the probability of an offspring conforming to type 1 behaviour is the probability that the offspring samples the majority of the parental generation performing type 1 behaviour, given the frequency of type 1 behaviour in the parental generation $\left(f_{A}+f_{S 1}\right)$. Thus, we sum across all possible samples of the majority (i.e. from $n / 2+1$ to the maximum sample size $n$ ) to give a stochastic model for the probability of conforming, weighted by $D$, where $1 \geq D>0$. For anti-conformists, the probability of adopting type 1 behaviour is negatively related to the probability of sampling type 1 behaviour in the majority, so $-1 \leq D<0$ (Figure 1 ). Figure 1c shows that the population size has a positive effect on the probability of conforming when $\left(f_{A}+f_{S 1}\right)>1 / 2$ and an equivalent negative effect when $\left(f_{A}+f_{S 1}\right)<1 / 2$, assuming $n$ is an odd number (the reverse pattern for non-conformists is not shown).

To examine the evolutionary dynamics analytically, we take the simple case where $n=3$ and this should be assumed in the results unless specified otherwise. As shown by Boyd and Richerson (1985), the probability of social learning under frequency-dependent bias when $n=3$ is

$$
\phi=\left(f_{A}+f_{S 1}\right)+D\left(f_{A}+f_{S 1}\right)\left[1-\left(f_{A}+f_{S 1}\right)\right]\left[2\left(f_{A}+f_{S 1}\right)-1\right]
$$

where $s=1$.

The fitness of asocial learners and social learners is

$$
W_{A}=1-c_{A}+b
$$


and

$$
W_{S}=1-c_{S}+b \frac{f_{S 1}}{f_{S 1}+f_{S 0}},
$$

respectively. Clearly, the fitness of social learners is a function of the proportion that receive the benefits of type 1 behaviour. For our analysis, we shall find it useful to re-express this proportion as the probability of a social learner adopting type 1 behaviour from the parental generation (superscript 'p'), so that

$$
W_{S}=1-c_{S}+b(1-e) s\left(f_{A}^{p}+f_{S 1}^{p}\right)\left[1+D\left(1-\left(f_{A}^{p}+f_{S 1}^{p}\right)\right)\left(2\left(f_{A}^{p}+f_{S 1}^{p}\right)-1\right)\right],
$$

where, for $D \neq 0, n=3$.

\section{Results}

\subsection{Evolutionary Trajectories}

\subsubsection{Unbiased Social Learning, $D=0$}

For any set of valid conditions, there are either two or three equilibria, $\hat{f}_{A}=1$, $\hat{f}_{S 0}=1$ and a genetically-polymorphic equilibrium

$$
\hat{f}_{S}=\frac{c_{A}-c_{S}-b[1-s(1-e)]}{s\left(c_{A}-c_{S}\right)(1-e)} .
$$

The genetically-polymorphic equilibrium occurs if, simultaneously, $W_{S}>W_{A}$

when $\hat{f}_{A}=f_{A}^{p}=1$, and $W_{A}>W_{S}$ when $\hat{f}_{S 0}=1$, which from (8) and (6), are 
both satisfied when

$$
c_{A}-c_{S}>b[1-s(1-e)]
$$

and

$$
b>c_{A}-c_{S},
$$

respectively (figure 2). The equilibrium (9) is locally stable where both $f_{S}>0$ and $f_{A}>0$ (see Figure $4 \mathrm{a}$ ).

If (10) is not satisfied, social learners cannot invade a population of asocial learners and the equilibrium $\hat{f}_{A}=1$ is locally stable, for $f_{A}>0$. Conversely, if (11) is not satisfied, asocial learners are driven out of the population and the absence of information producers results in the equilibrium $\hat{f}_{S 0}=1$, which is locally stable for $f_{S}>0$ (but note that $\hat{W}<1$ so the population goes extinct).

\subsubsection{Payoff-biased Social Learning, $s_{P B}=b$}

The conditions for the invasion of social learners in a population of asocial learners is given by inequality (10) and is a quadratic function of $b$. Figure $2 b$ shows that across a range of values of $e$, a genetically-polymorphic equilibrium may occur for small or large, but not intermediate values of $b$. Like unbiased social learners, the curve declines with $b$ when $b$ is small as the relative payoff to asocial learners increases. Unlike unbiased social learners, for larger values of $b$, the curve increases as the relative payoff effect is offset by the positive effect of $b$ on the probability of social learning type 1 behaviour. Note that a genetically-polymorphic equilibrium can occur under a greater values of $e$ than for unbiased social learning (or frequency-dependent social learning) if 
$b>s$ and thus $s_{P B}>s$, where $s$ is the fidelity of social transmission for unbiased (or frequency-dependent) social learning.

\subsubsection{Frequency-dependent Biased Social Learning, $-1 \leq D \leq 1, D \neq 0$}

There can be up to five equilibria for a single set of conditions (see Figure 4): $\hat{f}_{A}=1, \hat{f}_{S 0}=1$, a genetically-polymorphic equilibrium $0<\hat{f}_{S}<1$, and two culturally-polymorphic equilibria on the triangle boundary $f_{S}=1$ where $0 \leq f_{S 1} \leq 1$, defined by

$$
\hat{f}_{S 1}=\frac{3}{4}+\frac{\sqrt{D(1-e)-8 e}}{4 \sqrt{D} \sqrt{1-e}}
$$

and

$$
\hat{f}_{S 1}=\frac{3}{4}-\frac{\sqrt{D(1-e)-8 e}}{4 \sqrt{D} \sqrt{1-e}} .
$$

Both (12) and (13) exist if the degree of conformity $D>8 e /(1-e)$, where $e<1 / 9$. In contrast, the equilibrium at (13), but not (12), occurs for anticonformists that exhibit a weighting $D<-e /(1-e)$, where $e<1 / 2$.

Figure 3 shows conditions for distinct combinations of the equilibria where $\hat{f}_{S}>0$, for conformists, where $D=1$, and anti-conformists, where $D=-1$. Figure 4(b-i) shows examples of evolutionary trajectories from each region. All regions (apart from (IV)) satisfy the inequality (10), which is where $W_{S}>W_{A}$ at $f_{A}=1$, and result in an equilibrium, $\hat{f}_{S 1}>0$ that is locally stable where both $f_{S}>0$ and $f_{A}>0$. The equilibria, $\hat{f}_{A}=1$ and $\hat{f}_{S 0}=1$, occur in all the regions. For conformists, the boundary equilibrium (12) is locally stable in regions (i) and (ii), while the genetically-polymorphic equilibrium $\left(0<\hat{f}_{S}<1\right)$ is locally stable in regions (iii) and (iv). For anti-conformists, the 
boundary equilibrium (13) is locally stable in region (I), while the geneticallypolymorphic equilibrium $\left(0<\hat{f}_{S}<1\right)$ is locally stable in regions (II) and (III). Details of each region are documented below.

\section{Conformist Bias}

Region (i): Equilibria (12) and (13) both exist, where (12) has the higher frequency of type 1 behaviour, and is locally stable where both $f_{S}>0$ and $f_{A}>0$, if $W_{S}>W_{A}$ at (12), which from (8) and (6), occurs in the bounded region (see Figure 3a-b)

$$
e<\frac{D\left(c_{A}-c_{S}\right)\left(b-2\left(c_{A}-c_{S}\right)\right)}{b^{2}+D\left(c_{A}-c_{S}\right)\left(b-2\left(c_{A}-c_{S}\right)\right)} .
$$

The other boundary equilibrium, (13) is unstable. Example trajectories are shown in Figure 4(b).

The frequency of type 1 behaviour at (12) is affected only by the probability of environmental change, with which it is negatively related, culminating in $\hat{f}_{S 1}=1$ when $e=0$. The two boundary equilibria, (12) and (13), get closer with greater values of $e$ and are unified at an unstable equilibrium $\hat{f}_{S 1}=3 / 4$ at the apex of the curved line, where

$$
e=D /(8+D)
$$

(i.e. $e=1 / 9$, where $D=1$ ) which delimits the maximum probability of environmental change for which (12) and (13) co-occur.

Region (ii): The $f_{S}=1$ boundary equilibria (12) and (13) both exist, as the region is bounded above by (15), in addition to an unstable genetically- 
polymorphic equilibrium, $0<\hat{f}_{S}<1$. In this region, $W_{S}>W_{A}$ at $(12)$, and is satisfied by an alternative condition to (14), requiring

$$
c_{A}-c_{S}>b / 4
$$

(e.g. $c_{A}-c_{S}>0.05$ in Figure 3a). Consequently, (12) is locally stable where both $f_{S}>0$ and $f_{A}>0$, and discounting the unstable equilibrium $0<\hat{f}_{S}<1$, while (13) is unstable. Example trajectories are shown in Figure 4(c).

Region (iii): There exists a genetically-polymorphic equilibrium and the two equilibria, (12) and (13). In this region, neither (14) nor (16) are satisfied, so $W_{A}>W_{S}$ at the boundary equilibrium (12). Consequently, the geneticallypolymorphic equilibrium is locally stable where both $f_{S}>0$ and $f_{A}>0$, and at this equilibrium, $\hat{f}_{S 1}+\hat{f}_{A}>1 / 2$. The $f_{S}=1$ boundary equilibrium (12) is locally stable to perturbation only along the $f_{S}=1$ boundary, where a boundary population that exceeds (13) moves towards (12). Example trajectories are shown in Figure 3(d).

Region (iv): Both asocial learners and conformists either evolve to a polymorphic equilibrium state or enter a limit-cycle. Both cases are locally stable where both $f_{S}>0$ and $f_{A}>0$, and the frequency of type 1 behaviour may either be higher or lower than one half. This region lies above line (15), so a population on the $f_{S}=1$ boundary evolves towards $\hat{f}_{S 0}=1$ where the mean fitness of $\bar{W}<1$ and the population goes extinct. Example trajectories are shown in Figure 4(e).

\section{Anti-conformist Bias}

Region (I): The $f_{S}=1$ boundary equilibrium (13) exists and is locally stable 
where both $f_{S}>0$ and $f_{A}>0$, as $W_{S}>W_{A}$ at (13), where (14) is satisfied. In this region, $c_{A}-c_{S}$ always exceeds that of region (i) for conformists, and when $c_{A}-c_{S}=b$, region (I) is bounded by $e=D /(D-1)$ (i.e. $e=1 / 2$ where $D=-1$, which is greater than the maximum probability of environmental change afforded within region (i). The equilibrium frequency of type 1 behaviour on the $f_{S}=1$ boundary is negatively related to $e$ and has a maximum of $\hat{f}_{S 1}=1 / 2$, where $e=0$. Example trajectories are shown in Figure $4(\mathrm{f})$.

Region (II): A genetically-polymorphic equilibrium, $0<\hat{f}_{S}<1$, exists and is locally stable under the line $e=1 / 2$, where both $f_{S}>0$ and $f_{A}>0$. At this equilibrium, the frequency of type 1 behaviour may either be higher or lower than one half. The $f_{S}=1$ boundary equilibrium (13) also exists and is stable to perturbation only within the $f_{S}=1$ boundary. In this region, (14) does not hold at (13), so a polymorphic population evolves towards $0<\hat{f}_{S}<1$. Example trajectories are shown in Figure $4(\mathrm{~g})$.

Region (III): There exists a locally stable genetically-polymorphic equilibrium, $0<\hat{f}_{S}<1$, above the line $e=1 / 2$, where both $f_{S}>0$ and $f_{A}>0$. At this equilibrium, the frequency of type 1 behaviour may either be higher or lower than one half. In this region, $e$ is always too high for an equilibrium on the $f_{S}=1$ boundary to exist (where, $f_{S 1}>0$ ). Example trajectories are shown in Figure 4(h).

Region (IV): The $f_{S}=1$ boundary equilibrium (13) exists and is stable to perturbation only within the $f_{S}=1$ boundary. In this region, (10) is not satisfied, so (13) cannot be locally stable, yet $e<1 / 2$ so a population on the $f_{S}=1$ boundary evolves towards $\hat{f}_{S 1}>0$, where $f_{S 1}>0$. Example trajectories are shown in Figure 4(i). 
Numerical analysis shows conditions where the evolution of either conformists or anti-conformists can result in limit-cycles and highly complex dynamics, where $\bar{W}>1$ (Figure $4 \mathrm{j}-\mathrm{n})$.

Figure 4(j) shows that conformists can evolve to a stable limit-cycle. This occurs for conditions found in region (iv) (Figure 3), just above the line (15) and where the predicted equilibrium frequency of type 1 behaviour $\hat{f}_{A}+\hat{f}_{S 1} \approx$ $1 / 2$ (i.e. where $\left.e \approx 2\left(c_{A}-c_{S}\right) / b-1\right)$. During the limit-cycle, when $f_{A}+f_{S 1}>$ $1 / 2$, conformists spread as their fitness exceeds that of asocial learners. This is accompanied by a reduction in asocial learners and the frequency of type 1 behaviour. When $f_{A}+f_{S 1}<1 / 2$, conformists prefer not to adopt type 1 behaviour, the frequency of S1 individuals crashes and their fitness falls below that of asocial learners. Asocial learners increase in frequency until type 1 behaviour is in the majority again, and so the cycle continues.

Figure $4(\mathrm{k}-\mathrm{n})$ show dynamics for anti-conformists $(D=-1)$, where the sample size observed in the parental generation $n>3$. Apart from $n$, the conditions in part (l) are from region (I) in Figure 3, and accordingly, the polymorphic population evolves to the $f_{S}=1$ boundary, reaching a limit-cycle where $0<f_{S 1}<1$. The conditions in Figure $4(\mathrm{k} \& \mathrm{~m})$, apart from $n$, come from region (II) of Figure 3, and accordingly, genetically-polymorphic populations evolve to genetically-polymorphic limit-cycles. Numerical analysis suggests that limit-cycles occur when $e$ is particularly small and $n$ is high, favouring an equilibrium frequency of type 1 behaviour $\hat{f}_{A}+\hat{f}_{S 1} \approx 1 / 2$ (predicted when $n=3$ ) and a higher probability of adopting behaviour type 1 when it is observed in the minority than in the majority (a characteristic in Figure 1(b) but not $1(\mathrm{a})$ ). Particularly high $n$ can result in extremely complex bounded cycles (Figure $4 \mathrm{~m} \& \mathrm{n}$ ). These results are intriguing as they rely on endoge- 
nous properties of the system, largely in the absence of change in the external environment (i.e. when $e$ is very small).

\subsection{Genetic Fitness}

Here, we compare the fitness of different social learning rules and infer conditions favouring the evolution of each. Figure 5 (a \& c) shows the change in fitness with the invasion and spread of different social learning rules in separate populations of asocial learners. From the social learning fitness expression (8), and given the same frequency of type 1 behaviour in separate populations, the fitness of conformists exceeds that of unbiased social learners when $f_{S 1}+f_{A}>1 / 2$ but is less than unbiased social learners when $f_{S 1}+f_{A}<1 / 2$, with the reverse true for anti-conformists. Accordingly in Figure 5 (a \& c), when social learners are rare and thus frequency of type 1 behaviour is high, the fitness of anti-conformists is always less than that of unbiased social learners (or conformists). At the genetically-polymorphic equilibrium (where the fitness line for each social learning rule meets the asocial learning line), the equilibrium frequency of conformists exceeds that of unbiased social learners (or anti-conformists) when $\hat{f}_{S 1}+\hat{f}_{A}>1 / 2$ (Figure $5(\mathrm{a})$ ). Conversely, the equilibrium frequency of anti-conformists exceeds that of unbiased social learners (or conformists) when $\hat{f}_{S 1}+\hat{f}_{A}<1 / 2$ (Figure 5c). This occurs under the dashed line in regions (II) and (III) of Figure 3, where

$$
e<\frac{2\left(c_{A}-c_{S}\right)}{b}-1
$$

Figure 5c shows that although unbiased social learners (or conformists) have a higher fitness than anti-conformists when rare, the fitness of unbiased so- 
cial learners (or conformists) at their genetically-polymorphic equilibrium frequency is exceeded by that of anti-conformists at the same frequency. However, the reverse is not true, as unbiased social learners (or conformists) have a lower fitness than that of anti-conformists at the anti-conformist geneticallypolymorphic equilibrium frequency. Thus, we predict that anti-conformists would successfully invade a population of unbiased social learners (or conformists) that are at equilibrium with asocial learners when $\hat{f}_{S 1}+\hat{f}_{A}<1 / 2$, but that the reverse evolutionary dynamic would not occur under these conditions. As this prediction is based on comparisons of the fitness of social learning rules evolving in separate populations of asocial learners, we undertook numerical simulations based on equations (1)-(3) including two types of social learning rules evolving in a population of asocial learners, where the normalizing factor, $\bar{W}$, was adjusted appropriately. The analysis confirmed that if conformists evolve to a polymorphic equilibrium with asocial learners where $\hat{f}_{S 1}+\hat{f}_{A}<1 / 2$, anti-conformists can then invade and spread to a polymorphic equilibrium with asocial learners to the exclusion of conformists. Also as predicted, the reverse evolutionary dynamic between conformists and anti-conformists held when $\hat{f}_{S 1}+\hat{f}_{A}>1 / 2$.

Under conditions that favour a polymorphic equilibrium between asocial learners and a frequency-dependent social learning rule, the strength of frequencydependent bias, $D$, clearly affects the equilibrium frequency of the social learning rule. Figure 5 (b \& d) show that there is typically a negative relationship between the equilibrium frequency of social learning and the frequency of type 1 behaviour. This qualitative result is not influenced by whether the frequency-dependent bias is positively (part b) or negatively (part d) related to the equilibrium frequency of the social learning rule. This finding is sim- 
ply a consequence of the equilibrium frequency of asocial learners, who are assumed always to acquire type 1 information.

We used a numerical ESS method to examine the evolutionarily stable strength of frequency-dependent bias, $\hat{D}$, in relation to the probability of environmental change. For each value of $e$, we searched for $\hat{D}$ for which the fitness at the genetically-polymorphic equilibrium $\left(\hat{W}_{A}\right)$ was not exceeded by that of any rare mutant with a different value of $D$. We found that when $\hat{f}_{S 1}+\hat{f}_{A}<1 / 2$, $\hat{D}=-1$ and when $\hat{f}_{S 1}+\hat{f}_{A}>1 / 2, \hat{D}=1$. Thus considering (17), there is a discrete positive relationship between $\hat{D}$ and the probability of environmental change such that in stable environments, anti-conformity is typically favoured, while in changeable environments, conformity is typically favoured. Note, this finding is for the case $n=3$ and thus frequency-dependent bias is relatively mild (see Figure 1).

Overall, these results suggest that over evolutionary time, punctuated by the repeated invasion of different social learning rules, we expect to find a continuous reduction in both the frequency of asocial learning and of type 1 behaviour. The fitness of a new social learning rule, that successfully invades a population residing at a genetically-polymorphic equilibrium, will decline as it increases in frequency until its fitness approaches the asocial learning fitness line to the right-hand side of the original equilibrium state (see Figure 5). The reduction in information-producers (asocial learners) is accompanied by a reduction in type 1 behaviour. In this respect, the findings suggest a general rule, conceptually equivalent to Tilman's $\mathrm{R}^{*}$ rule (Tilman, 1982), which specified that, among competitors for a resource, the dominant competitor will be the species than can persist at the lowest resource level. Similarly, we predict that among competing social learning rules, the fittest rule will be the one that can persist 
with the lowest frequency of asocial learning.

\section{Discussion}

We have presented a gene-culture coevolutionary analysis of the evolution of a small number of social learning rules in a fluctuating environment, and defined conditions under which each rule evolves to fixation or to a polymorphic equilibrium with asocial learners. The later equilbria can also be regarded as expectations for the frequencies of asocial and social learning in a population of mixed strategists. Our novel findings include some interesting dynamical properties of these rules, including limit cycles and chaotic behaviour, a general rule specifying which of a series of competing social learning rules will become dominant and evolve to replace the alternatives, and further conditions under which social learning will increase the mean fitness of individuals in the population at equilibrium.

With regard to unbiased social learning, our coevolutionary analysis reaches the same equilibrium as that derived using a non-coevolutionary approach applied by Enquist et al. (2007), where the cultural trait is assumed to reach equilibrium prior to genetic selection (their result assumes $s=1$ ). Moreover, our numerical analysis indicates an equivalent polymorphic equilibrium for unbiased social learners and asocial learners to the coevolutionary analysis of Feldman et al. (1996), but where the environment was assumed to change with certainty to a novel state at a rate $1 / e$ (i.e. an infinite states environment). This confirms the generality of these earlier findings.

In our analysis, we explored the relative utility of reliance on pure asocial and 
social learning strategies, with increases in one strategy leading to a corresponding decrease in the other. However, we do not interpret this as necessarily implying that there is an evolutionary tradeoff in social and asocial learning capabilities. To the contrary, the observed propensity for social learning and behavioural innovation (which is largely asocial learning) covaries strongly across non-human primates (Reader and Laland (2002)), and we expect this finding to apply generally. Rather, we interpret our findings as indicating regions of the parameter space in which reliance on social or asocial learning rules will differ in their utility. While learning models that deploy a mix of social and asocial learning, like analyses of pure strategies, often assume a tradeoff in use of social and asocial learning (Boyd and Richerson (1985)), such a tradeoff within species is not inherently incompatible with the observation of covariation in these capabilities between species.

Previous analyses have found conditions where social learning rules can evolve to an ESS where the mean fitness of individuals is higher than that in an exclusively asocial learning population (Boyd and Richerson, 1995; Kameda and Nakanishi, 2003). These rules require cognitive flexibility, such that individuals switch between asocial and social learning (Boyd and Richerson, 1995; Kameda and Nakanishi, 2003; Efferson et al., 2008). To our knowledge, this increment in fitness at equilibrium has not previously been shown for a pure social learning rule (frequency-dependent bias) and provides another mechanism by which the evolution of social learning may result in population expansion. The result requires that the probability of environmental change is low, yet we might anticipate rare environmental catastrophies (e.g. $e=1$ ), whereupon type 1 behaviour would be lost $\left(\hat{f}_{S 0}=1\right)$ and the population size would decline $(\bar{W}<1)$. However, $\hat{f}_{S 0}=1$ is unstable and susceptible to invasion by asocial 
learners, so the population will always evolve back to the original equilibrium on the $f_{S}=1$ boundary.

Our analysis confirms the findings of Wakano and Aoki (2007) and Nakahashi (2007), of a negative relationship between the evolutionarily stable strength of frequency-dependent bias and environmental stability, and extends it to include anti-conformist biases. We find that $\hat{D}$ switches between anti-conformism and conformism as the frequency of type 1 behaviour at the polymorphic equilibrium, $\hat{f}_{S 1}+\hat{f}_{A}$, exceeds one half, and this occurs with a reduction in environmental stability (i.e. favouring asocial learners).

Efferson et al. (2008) observed that in a constant environment, conformity, but not anti-conformity, can result in homogeneity of behaviour as a bias to adopt a behaviour that is in the majority drives that behaviour to fixation. They also showed that strong anti-conformity can result in oscillations as the behaviour in the minority is always preferred and thus is driven into the majority, where the same behaviour is rejected with a high probability. In contrast, our geneculture coevolutionary time-frame in a variable environment reveals oscillatory behaviour for conformist bias as well as anti-conformist bias. We find that there can be not only stable culturally-polymorphic equilibria (i.e. variation in behaviour), but also stable limit cycles, where asocial learning fitness is greater than conformist fitness when type 1 behaviour is in the majority but not when in the minority.

The oscillations reported by Efferson et al. (2008) under conditions of 'strong' anti-conformity, occured when the frequency of the learned behaviour oscillates around a pivot of one half with sequential time steps. Our coevolutionary time-scale results in limit cycles that can oscillate at a much slower rate 
(e.g. Figure $4 \mathrm{j}: \approx 60$ generations per cycle). Also, Efferson et al's definition of 'strong' anti-conformity assumes that the probability of social learning curve is the mirror image of curves for conformist transmission, reflected in the line $f_{A}+f_{S 1}=1 / 2$ (i.e. the probability tends to one as the frequency of behaviour tends to zero). In contrast, we consider the case where $n$ is high, such that the effect of anti-conformity is enhanced, although there is a low social learning probability when type 1 behaviour is extremely rare. This makes biological sense, as an anti-conformist individual is unlikely to observe, and thus learn, a very rare type 1 behaviour if the population is large. We anticipate that the chaotic dynamics that we found are caused by the non-monotonic shape of the probability of social learning curve when the demonstrator sample size $n$ is high (see Figure 1).

For conditions favouring the fixation of frequency-dependent biased social learners, our derived equilibrium frequency of type 1 behaviour is consistent with Efferson et al. (2008), who predicted that conformity, but not anticonformity, can result in homogeneity of (type 1) behaviour. However, we also found that for both conformist and anti-conformist evolution, type 1 behaviour can be either in the majority or the minority at a genetically-polymorphic equilibrium.

The payoff-biased rule is a form of a more general 'copy if better' rule, which requires that social learners only copy a behaviour if the payoff is greater than that of their current behaviour (Schlag, 1998; Laland, 2004). Our implementation is a simple version of Schlag's (1998) proportional imitation rule, restricted to a single learned behaviour. While Schlag found this rule to be optimal, we suggest that in an evolutionary context, the success of the rule may be bound by the probability of environmental change in a concave pattern 
with respect to payoff (see Figure $2 b$ ). From a biological perspective, the payoff is having two separate effects on the conditions for invasion by payoff-biased social learners. First, asocial learners make up a greater proportion of the population receiving a payoff $(b)$ as the probability of environmental change increases. This differential proportion is equivalent to a finder's advantage in a producer-scrounger model, but one that is a positive function of the probability of environmental change. If the payoff of type 1 behaviour gets larger, the probability of environmental change must become smaller for payoff-biased social learners to invade. The same relationship also holds for unbiased and frequency-dependent social learners. Second, for particularly high payoffs from type 1 behaviour, the positive effect of observed payoff on the fidelity of social learning allows payoff-biased social learning to evolve in more variable environments as the payoff gets larger. This relationship is not observed for unbiased or frequency-dependent social learning, and potentially, can be used in empirical studies to help distinguish payoff-biased social learning.

We predict that the evolution of social learning rules should resemble Tilman's $R^{*}$ rule, such that the fittest rule will the one that can persist with the lowest frequency of asocial learning (Tilman, 1982). Thus, our results are consistent with the expectation that social learning rules become more efficient over evolutionary time, that is, they require fewer asocial learners (or less asocial learning) with time. This may help to explain our species' extraordinary reliance on culture. 


\section{Acknowledgements}

Research supported by a BBSRC grant to KNL and a RCUK fellowship to JRK. We also thank an anonymous reviewer for their helpful comments.

\section{References}

Aoki, K., Wakano, J., Feldman, M., 2005. The emergence of social learning in a temporally changing environment: A theoretical model. Curr. Anthrop. $46,334-340$.

Barnard, C., Sibly, R., 1981. Producers and scroungers: a general model and its application to captive flocks of house sparrows. Anim. Behav. 29, 543-555.

Boyd, R., Richerson, P., 1985. Culture and the Evolutionary Process. Chicago University Press.

Boyd, R., Richerson, P., 1995. Why does culture increase human adaptability? Ethol. Sociobiol. 16, 125-143.

Cavalli-Sforza, L., Feldman, M., 1981. Cultural Transmission and Evolution: A Quantitative Approach. Princeton University Press.

Coolen, I., Bergen, Y., Day, R., Laland, K., 2003. Species difference in adaptive use of public information in sticklebacks. Proc. Roy. Soc. Lond. B: Biol. Sci. 270 (1531), 1413-1419.

Efferson, C., Lalive, R., Richerson, P., McElreath, R., Lubell, M., 2008. Conformists and mavericks: the empirics of frequency-dependent cultural transmission. Evol. Human Behav. 29, 56-64.

Enquist, M., Eriksson, K., Ghirlanda, S., 2007. Critical points in current theory of conformist social learning critical points in current theory of conformist social learning. J. Evol. Psychol. 5 (67-87). 
Feldman, M., Aoki, K., Kumm, J., 1996. Individual versus social learning: evolutionary analysis in a fluctuating environment. Anthropol. Sci. 104 (3), 209-231.

Galef, B., Giraldeau, L.-A., 2001. Social influences on foraging in vertebrates: causal mechanisms and adaptive functions. Anim. Behav. 61, 3-15.

Galef, B., Whiskin, E., 2008. 'conformity' in rats? Anim. Behav. 75, 20352039.

Giraldeau, L.-A., Caraco, T., 2000. Social foraging theory. Princeton, NJ: Princeton University Press.

Giraldeau, L.-A., Lefebvre, L., 1987. Scrounging prevents cultural transmission of food-finding behaviour in pigeons. Anim. Behav. 35, 387-394.

Giraldeau, L. A., Valone, T., Templeton, J., 2002. Potential disadvantages of using socially acquired information. Philos. Trans. R. Soc. Lond. B Biol. Sci. 357, 1559-1566.

Henrich, J., Boyd, R., 1998. The evolution of conformist transmission and the emergence of between-group differences. Evol. Human Behav. 19, 215-241.

Henrich, J., McElreath, R., 2003. The evolution of cultural evolution. Evol. Anthropol. 12 (3), 123-135.

Kameda, T., Nakanishi, D., 2002. Cost-benefit analysis of social/cultural learning in a non-stationary uncertain environment: An evolutionary simulation and an experiment with human subjects. Evol. Human Behav. 23, 373-393.

Kameda, T., Nakanishi, D., 2003. Does social/cultural learning increase human adaptability? rogers's question revisited. Evol. Human Behav. 24, 242-260.

Kendal, R., Coolen, I., Bergen, Y., Laland, K., 2005. Tradeoffs in the adatptive use of social and asocial learning. Adv. Study Beh. 35, 333-379.

Kingsolver, J., Hoekstra, H., Hoekstra, J., Berrigan, D., Vignieri, S., Hill, C., Hoang, A., Gilbert, P., Beerli, P., 2001. The strength of phenotypic selection 
in natural populations. Am. Nat. 157, 245-261.

Laland, K., 2004. Social learning strategies. Learn. Behav. 32 (1), 4-14.

Nakahashi, W., 2007. The evolution of conformist transmission in social learning when the environment changes periodically. Theor. Popul. Biol. 72, 5266.

Reader, S., Laland, K., 2002. Social intelligence, innovation, and enhanced brain size in primates. Proc. Natl. Acad. Sci. USA 99, 4436-4441.

Rogers, A., 1988. Does biology constrain culture? Am. Anthrop. 90 (819-831).

Schlag, K., 1998. Why imitate, and if so, how? J. Econ. Theory 78, 130-156.

Tilman, D., 1982. Resource competition and community structure. Princeton, NJ: Princeton University Press.

Wakano, J., Aoki, K., 2007. Do social learning and conformist bias coevolve? henrich and boyd revisited. Theor. Popul. Biol. 72, 504-512.

Whiten, A., Ham, R., 1992. On the nature and evolution of imitation in the animal kingdom: reappraisal of a century of research. Adv. Study Beh. 21, $239-283$. 


\section{Legends}

Figure 1: The probability of social learning behaviour type 1 as defined by (4) and in relation to the frequency of the behaviour in the parental generation, where frequency-dependent social learners conform or anti-conform with a weighting $D$ where (a) $n=3$, (b) (b) $n=17$ and (c) conform upon sampling the behaviour of $n$ individuals in the parental generation where $D=1$. In all cases, $s=1$.

Figure 2: Under the each line, there exists a genetically-polymorphic equilibrium between asocial learners and either unbiased social learners (continuous line), or payoff-biased social learners (dashed line), satisfying inequalities (10) and (11). In (a) $c_{A}$ varies and $c_{S}=0.01$ while in part (b) $b$ varies. Otherwise, $D=0, c_{A}=0.27, c_{S}=0.01, b=0.5$.

Figure 3: Regions of parameter space that result in distinct combinations of equilibria when plotting $e$ against (a \& c) the difference in the cost of asocial learning and frequency-dependent social learning, $c_{A}-c_{S}$, where $c_{A}$ varies and $c_{S}=0.01$, and $(\mathrm{b} \& \mathrm{~d})$ the benefit $b$ of type 1 behaviour. In parts (a) and (b), frequency dependent social learners are conformists, $D=1$, and in parts (c) and (d), frequency dependent social learners are anti-conformists, $D=-1$. At genetically-polymorphic equilibria, the frequency of type 1 be-

haviour $\hat{f}_{A}+\hat{f}_{S 1}<1 / 2$ below the dashed line (inequality (17)); this line does not demark region boundaries. In part (a), 'L-Cycles' refers to limit-cycles. Unless specified otherwise, $c_{S}=0.01, c_{A}=0.07, b=0.2, s=1, n=3$. 
Figure 4: The triangular diagrams plot the evolutionary trajectories (arrows) of the frequency of asocial learners (A), social learners with type 1 behaviour (S1) and social learners without type 1 behaviour (S0). Only one state exists in each corner. The black disc represents a globally stable equilibrium, assuming $f_{A}>0$ and $f_{S}>0$, and the white discs indicate unstable equilibria. In part (a) social learners are unbiased and $D=0, c_{A}=0.04, c_{S}=$ $0.01, b=0.2, e=0.105, s=1$. In parts $(\mathrm{b}-\mathrm{i})$ social learners are frequencydependent and show trajectories from each region defined in Figure 3 . In (b) $D=1, c_{A}=0.05$, (c) $D=1, c_{A}=0.12$, (d) $D=1, c_{A}=0.04$ (e) $D=1, c_{A}=0.18, e=0.2$, (f) $D=-1, c_{A}=0.13$, (g) $D=-1, c_{A}=0.12$, (h) $D=-1, c_{A}=0.14, e=0.51$ and (i) $D=-1, c_{A}=0.07, e=0.2, b=0.45$, where $c_{S}=0.01, b=0.2, e=0.105, s=1, n=3$ unless stated otherwise. Parts $(\mathrm{j}-\mathrm{n})$ show oscillatory evolutionary trajectories where $c_{S}=0.01, b=0.2$ and in part $(\mathrm{j}) D=1, c_{A}=0.1, e=0.17, n=3,(\mathrm{k}) D=-1, c_{A}=$ 0.07, $e=0.001, n=17$, (l) $D=-1, c_{A}=0.15, e=0.001, n=17$, and (m) $D=-1, c_{A}=0.07, e=0.001, n=501$. Part $(\mathrm{n})$ shows dynamics of $\mathrm{S} 1$ (continuous line) and A (dashed line) over a generational timescale, where $D=-1, c_{A}=0.15, e=0.001, n=501$.

Figure 5: Parts (a) and (c) show the evolutionary trajectories (arrows) of genetic fitness $(6,7)$ of unbiased social learners (grey line, $D=0$ ), conformists (continuous black line, $D=1$ ) and anti-conformists (dashed black line, $D=-1$ ) following invasion in separate populations of asocial learners (thick black line). In each case, the equilibrium frequency of the social learning rule is where its line meets the asocial learning line. Parts (b) and (d) show the relationship between the equilibrium frequency of social learners $\left(\hat{f}_{S}\right)$ and the equilibrium frequency of type 1 information $\left(\hat{f}_{A}+\hat{f}_{S 1}\right)$ plot- 
ted from simulations where $-1 \leq D \leq 1$. In parts (a) and(b), $e=0.55$, $\hat{f}_{A}+\hat{f}_{S 1}>1 / 2$, and $D$ is positively associated with $\hat{f}_{S}$. In parts (c) and (d), $e=0.35, \hat{f}_{A}+\hat{f}_{S 1}<1 / 2$ and $D$ is negatively associated with $\hat{f}_{S}$. All populations start at $\left(f_{A}=0.99, f_{S 0}=0.01\right)$, and other parameter values are $c_{A}=0.155, c_{S}=0.01, b=0.2, s=1, n=3$.

Figure 6: The fitness at equilibrium in a population of only (a) conformists and (b) anti-conformists (thin lines) and, for comparison, the fitness of asocial learners (thick lines), across a range of values for the probability of environmental change, $e$, where conditions favour the evolution of frequencydependent social learners to fixation, where (a) $D=1, c_{A}=0.05$ (see Figure 3a, Region (i)) and (b) $D=-1, c_{A}=0.17$ (see Figure 3c, Region (I)) . Other parameter values are $c_{S}=0.01, b=0.2, s=1, n=3$. 

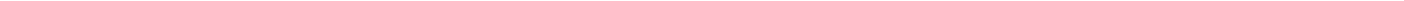


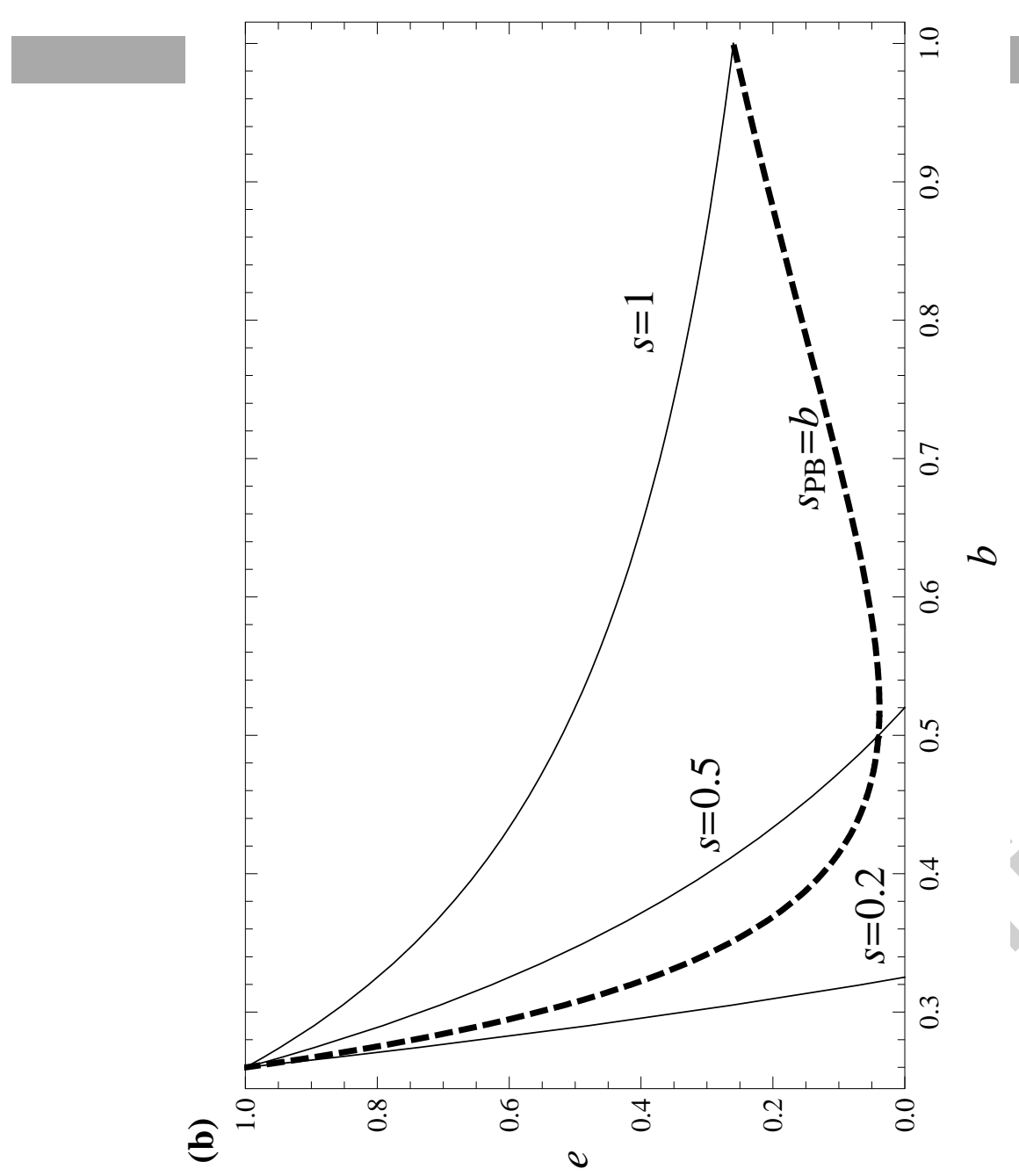

N
ํㅣㄴ
은

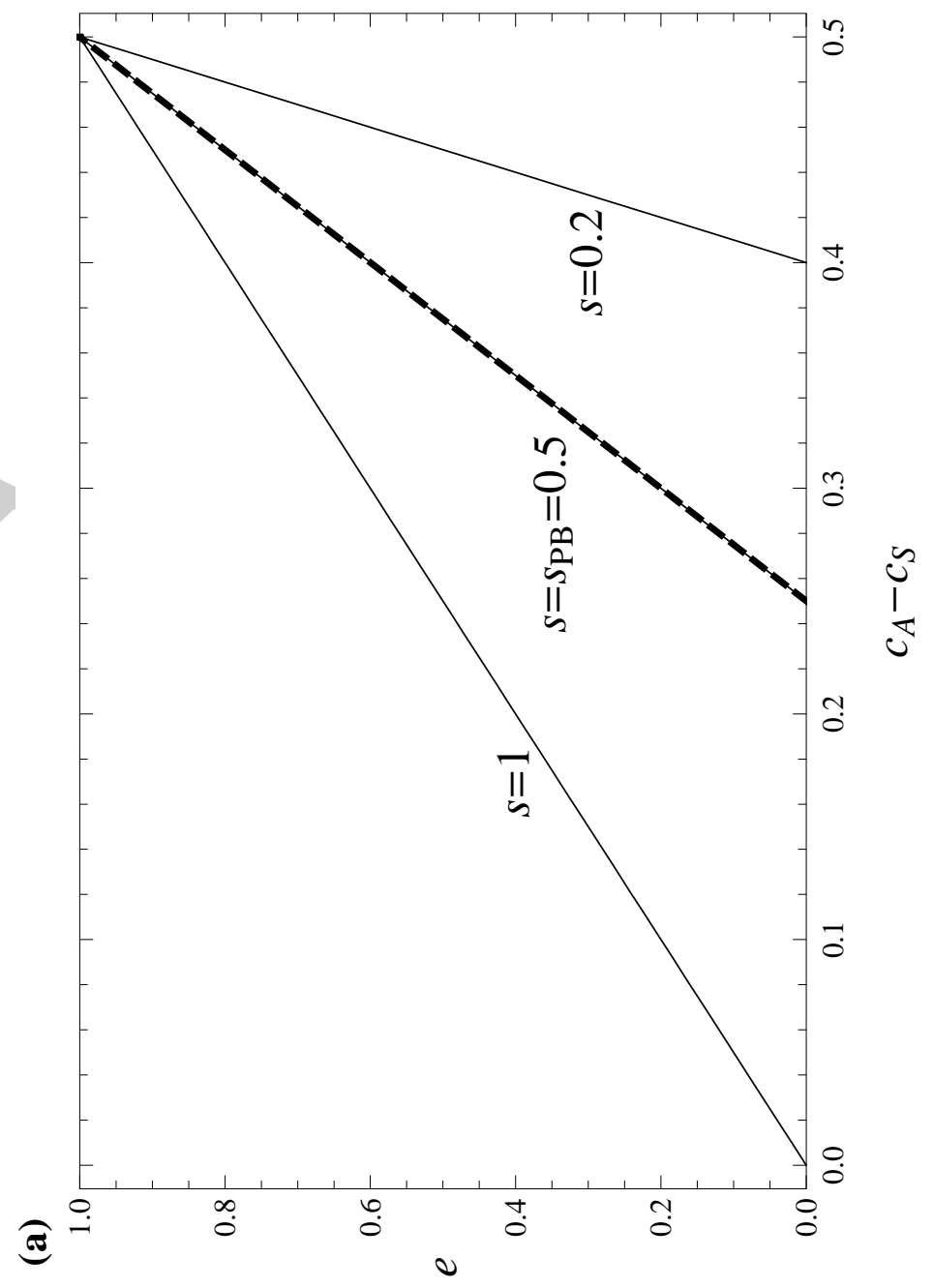



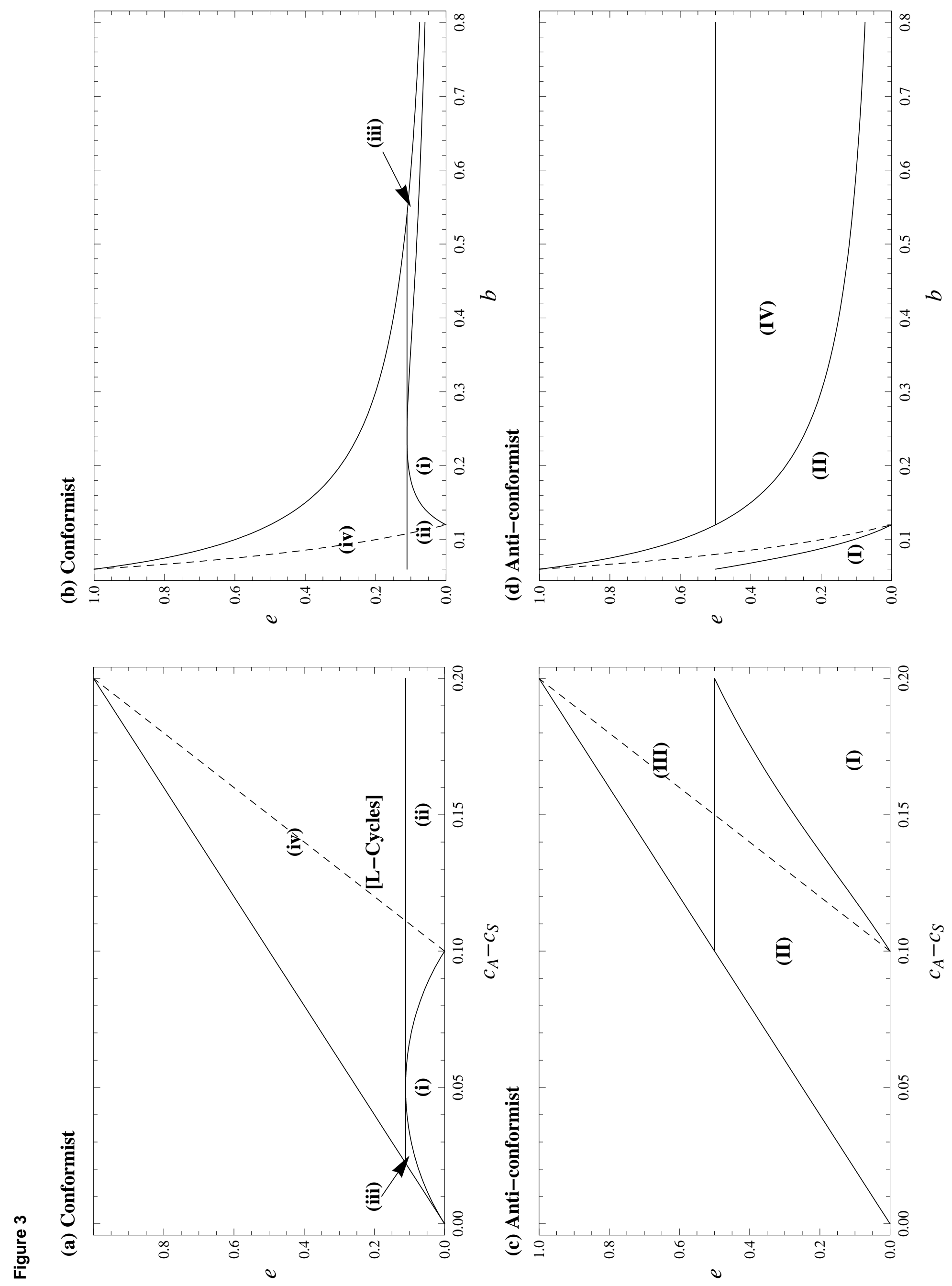
(a) Unbiased

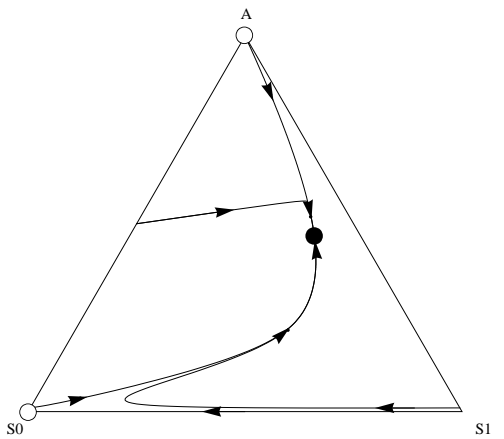

(d) Conformist, region (iii)

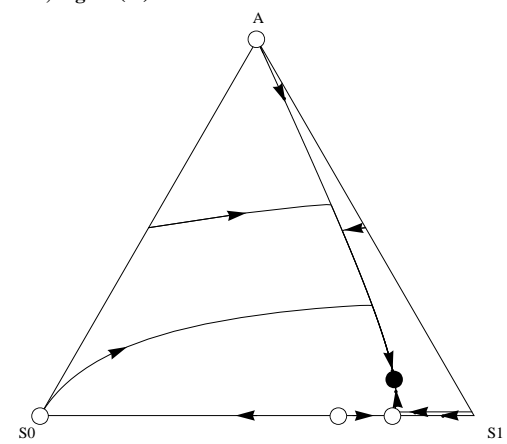

(g) Anti-conformist, region (II)

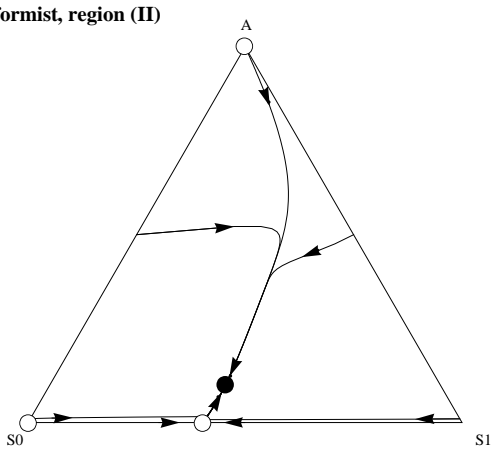

(j) Conformist, $n=3$

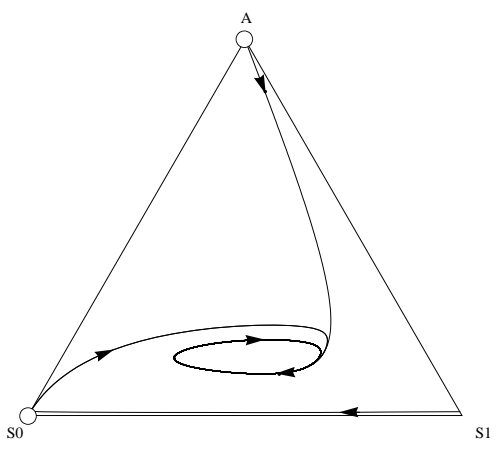

(m) Anti-conformist, n=501

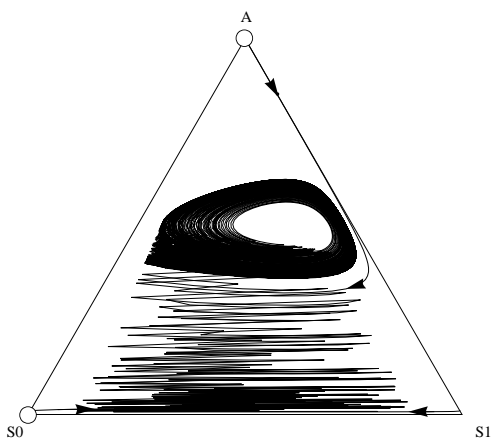

(b) Conformist, region (i)

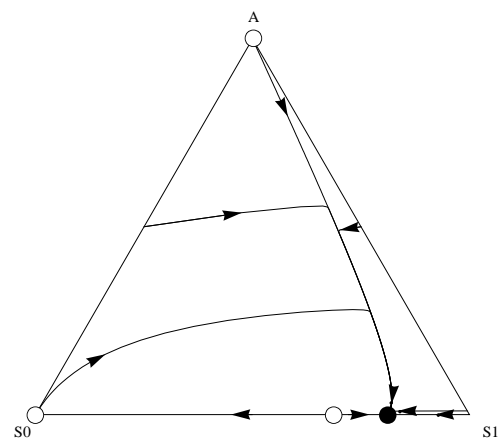

(e) Conformist, region (iv)

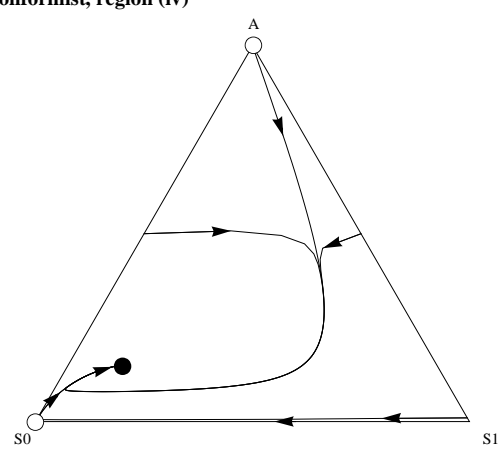

(h) Anti-conformist, region (III)

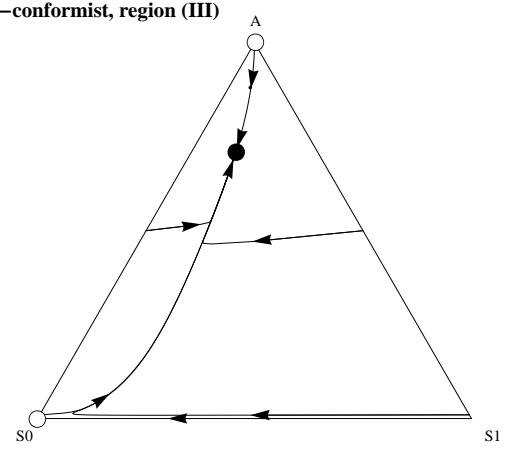

(k) Anti-conformist, n=17

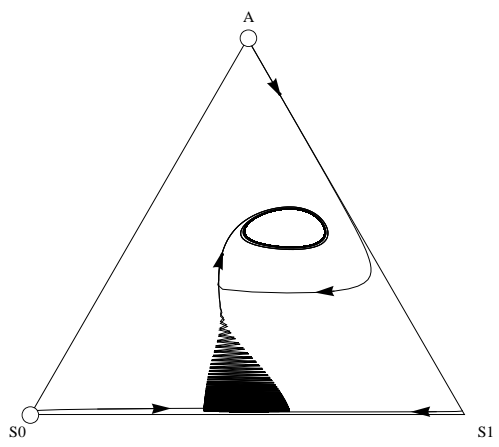

(n) Anti-conformist, $\mathrm{n}=501$

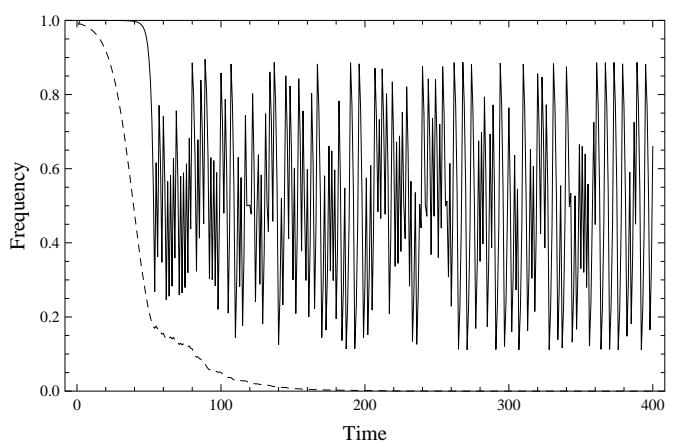

(c) Conformist, region (ii)

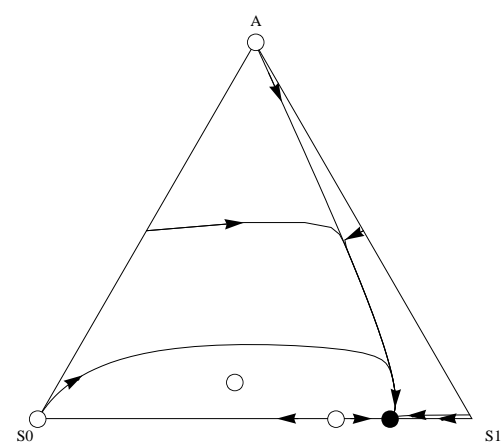

(f) Anti-conformist, region (I)

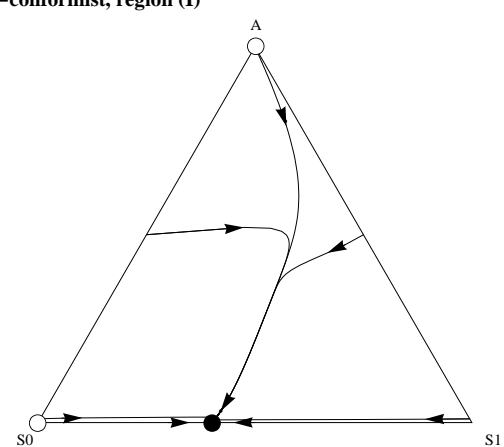

(i) Anti-conformist, region (IV)

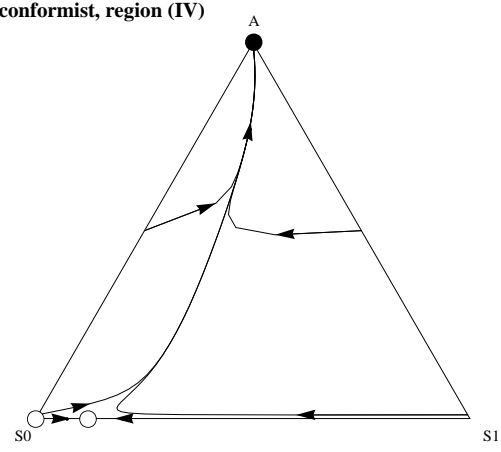

(l) Anti-conformist, $\mathrm{n}=17$

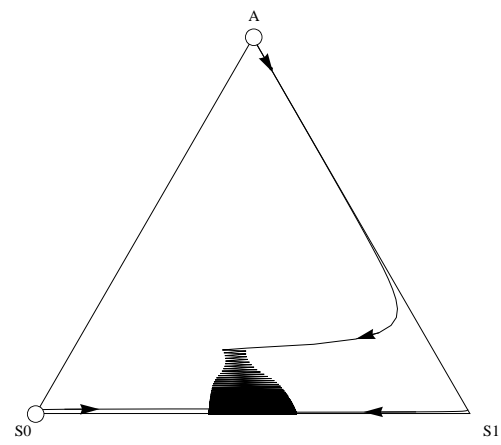



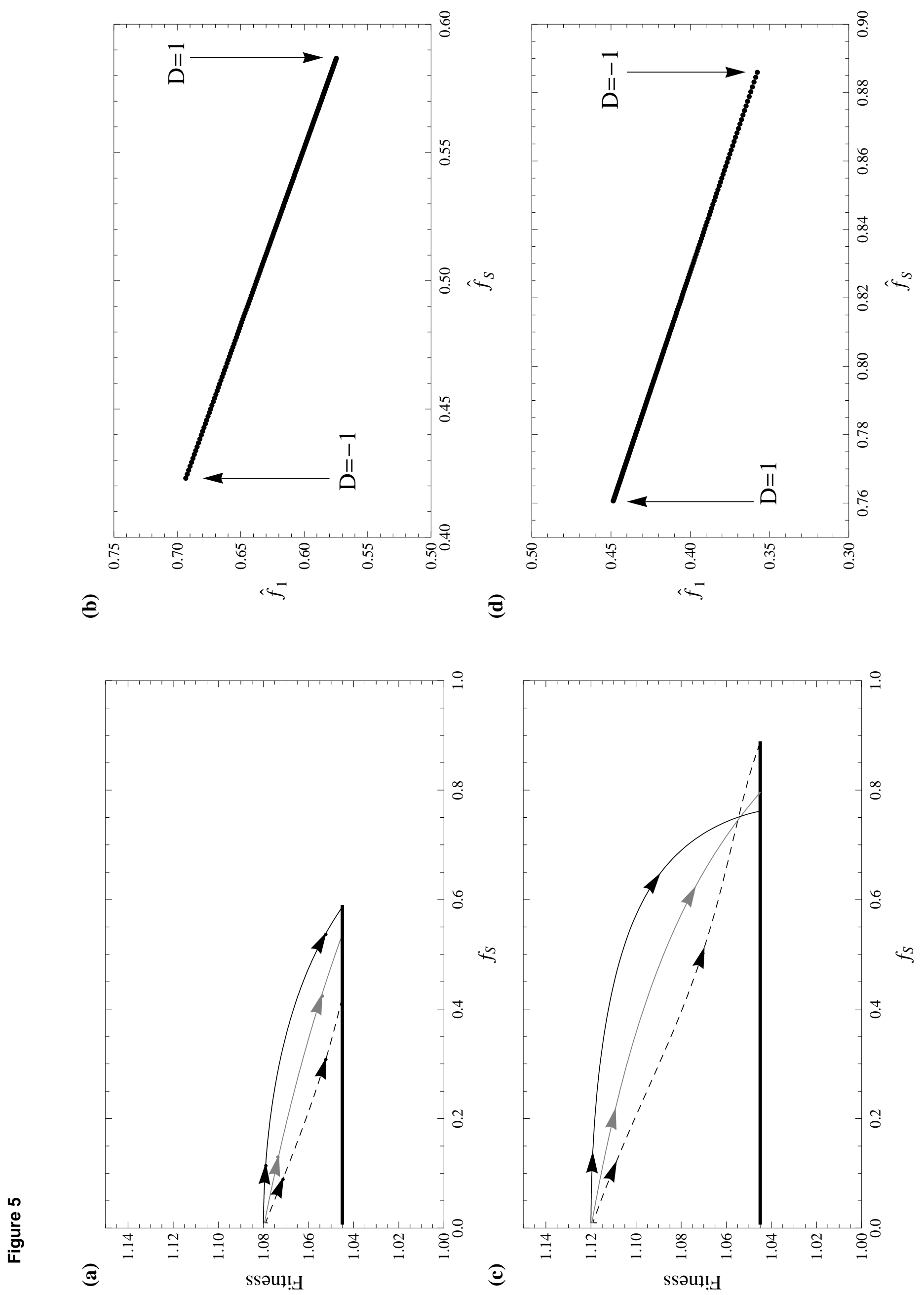


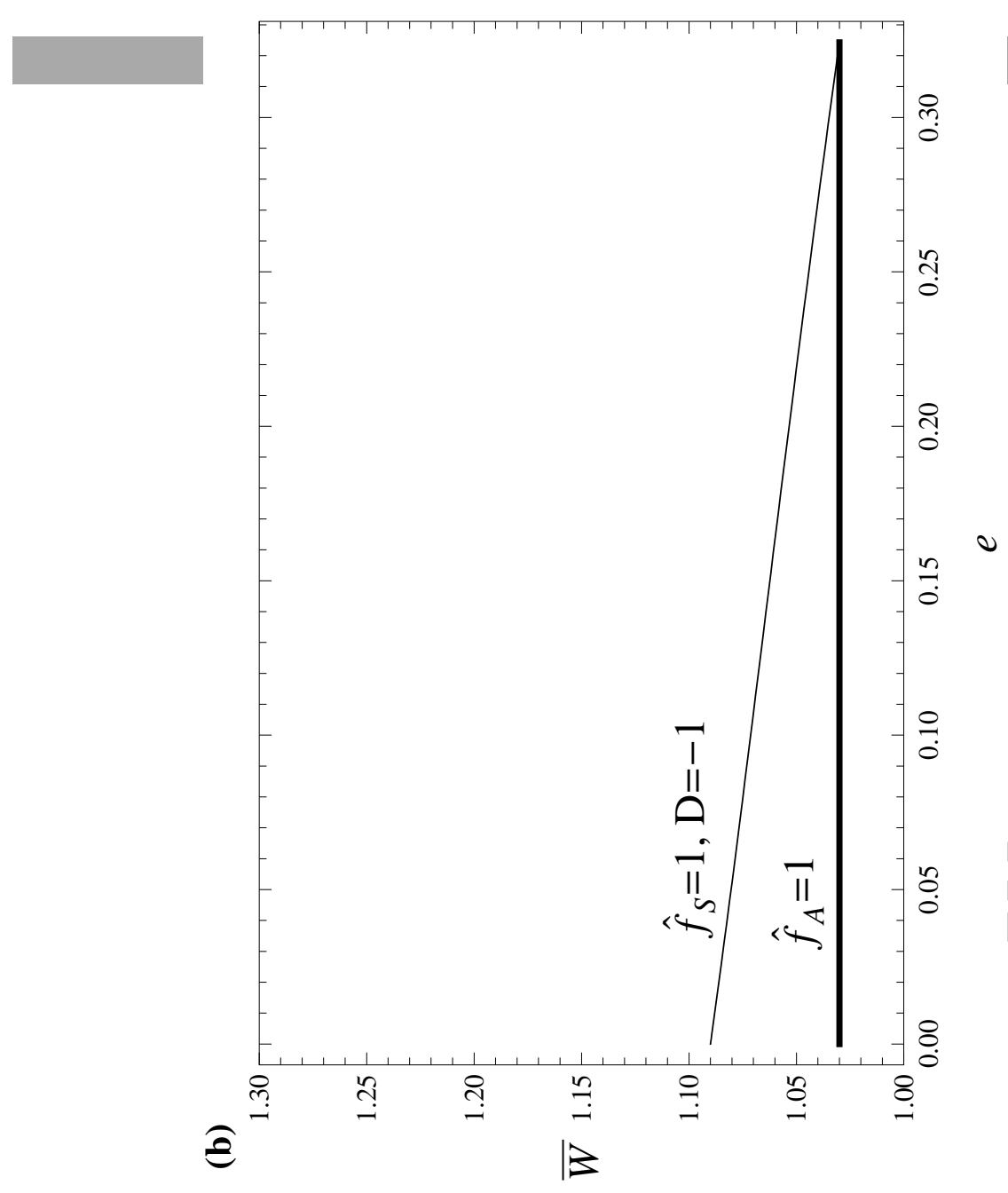

0
0
는
문

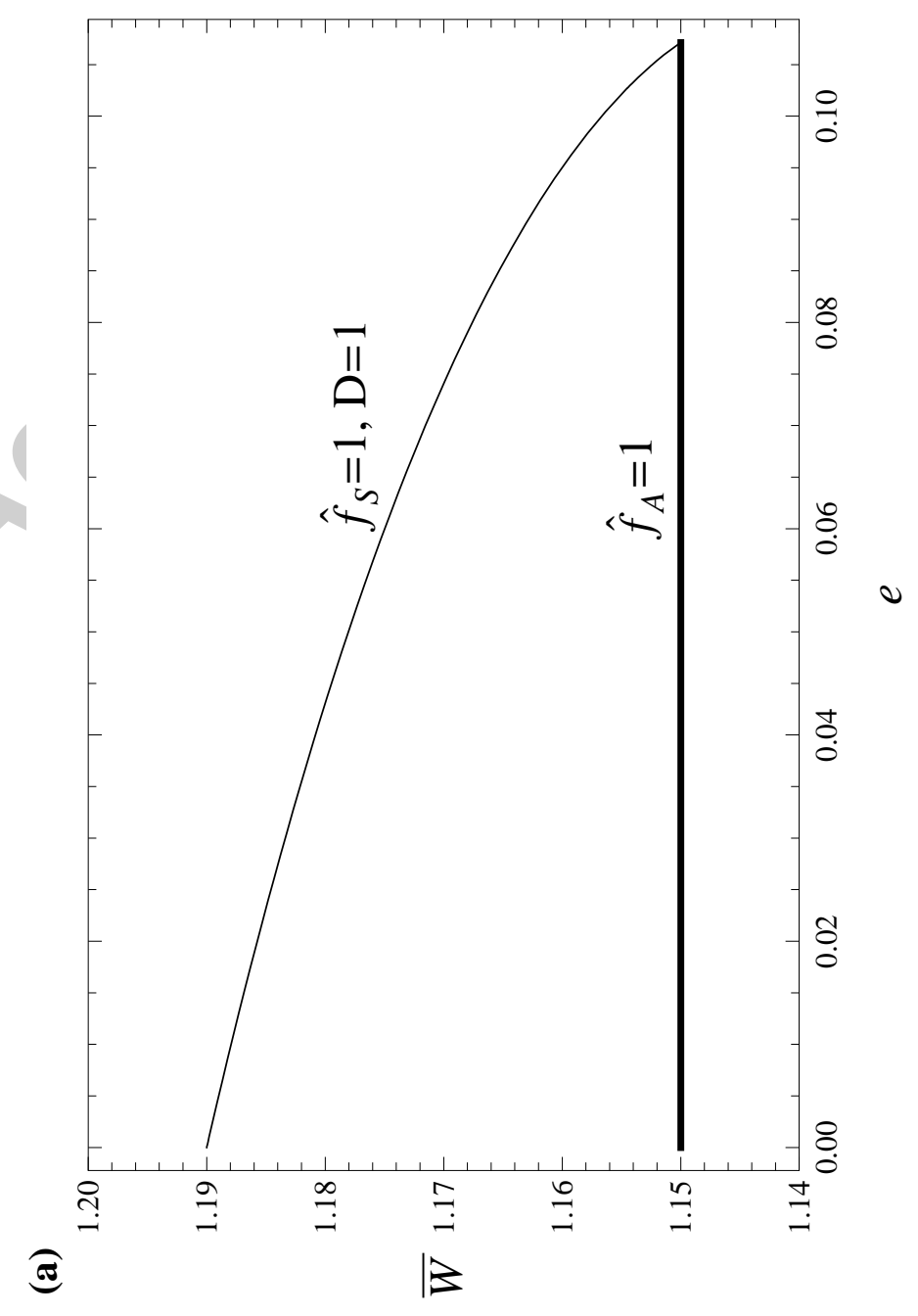

